\title{
Risk Factors for Moderate-to-severe Postoperative Pain After Percutaneous Nephrolithotomy:A Retrospective Study
}

Hao-Tian Wu

Beijing Tsinghua Changgung Hospital

Tian-fu Ding

Beijing Tsinghua Changgung Hospital

Huan Zhang ( $\nabla$ whta01956@btch.edu.cn )

Beijing Tsinghua Changgung Hospital

\section{Research Article}

Keywords: percutaneous nephrolithotomy (PCNL),risk facto

Posted Date: April 13th, 2021

DOl: https://doi.org/10.21203/rs.3.rs-396652/v1

License: (c) (i) This work is licensed under a Creative Commons Attribution 4.0 International License.

Read Full License 


\title{
Risk factors for moderate-to-severe postoperative pain after percutaneous nephrolithotomy:A retrospective study
}

\author{
Hao-Tian Wu,Tian-fu Ding,Huan Zhang*
}

Department of Anesthesiology,Beijing Tsinghua Changgung Hospital,School of Clinical Medicine,Tsinghua University,Beijing 102218,China

*Corresponding author:Huan Zhang

Email: whta01956@btch.edu.cn

Department of Anesthesiology

Beijing Tsinghua Changgung Hospital

School of Clinical Medicine

Tsinghua University 


\section{Abstract}

Backgroud and Objectives: Percutaneous nephrolithotomy (PCNL) is a minimally invasive procedure for removing renal calculus, but a large number of patients experience acute moderate-to-severe pain despite the analgesia provided.The aim of this study is to explore the risk factors for postoperative pain after percutaneous nephrolithotomy (PCNL), and provides a reference for clinical prevention of postoperative pain after PCNL.

Methods: According to the inclusion and exclusion criteria, the clinical data of 331 patients who underwent PCNL in our hospital from September 2019 to February 2021 were retrospectively collected. General anesthesia was induced with propofol, sufentanil and rocuronium and maintained with sevoflurane, remifentanil and rocuronium.Depending on whether the patient had moderate-to-severe postoperative pain, they were divided into mild or no pain group and moderate-to-severe pain group. We collected and analyzed data on age,gender, body mass index (BMI),ASA grading,co-morbidities, number of renal calculus, renal calculus size, degree of hydronephrosis, preoperative urine culture,preoperative creatinine,stone burdens, expansion method,tract diameter, nephrostomy tube,calculi residual,operative position ,puncture time , number of expanded channels, expanded channel time, duration of surgery,vital signs and VAS pain score.The indicators with statistically significant differences were selected and multivariate logistic regression analysis was carried out to determine the risk factors for postoperative pain. 
Results: Among 331 patients, 221 had moderate-to-severe pain and the incidence rate was $66.77 \%$. Univariate analysis and multivariate logistic regression analysis showed that independent risk factors for moderate-to-severe pain after PCNL were:number of renal calculus(OR=5.303, $P=0.001)$,stone burdens (OR=7.623, $\mathrm{P}=0.001)$, calculi residual $(\mathrm{OR}=0.2377, \mathrm{P}=0.001)$, duration of surgery $(\mathrm{min})(\mathrm{OR}=1.024$, $\mathrm{P}=0.001)$.

Conclusions: The main risk factors for pain after PCNL are number of renal calculus, stone burdens, calculi residual and duration of surgery.

Keywords: percutaneous nephrolithotomy (PCNL), risk factors, pain

\section{Background}

Over the past two decades, open surgery has been almost completely replaced by minimally invasive procedures for patients with kidney stones ${ }^{1}$.According to the European Association of Urology guidelines, Percutaneous nephrolithotomy $(P C N L)$ is the recommended first-line treatment of large $(>2 \mathrm{~cm})$, multiple and inferior calyx renal stones PCNL is generally considered a safe technique offering the highest stone-free rates after the first treatment as compared to the other minimal invasive lithotripsy techniques ${ }^{2}$.

Unilateral percutaneous nephrolithotomy (PCNL) is the surgical procedure of choice for large, multiple or 
complex kidney stones. It is often associated with severe postoperative somatosensory and visceral pain, necessitating a substantial requirement for postoperative opioid consumption ${ }^{3}$.Pain after PCNL included creation of a percutaneous access tract through the parenchyma and parenchymal shearing, renal pelvic pressure (RPP), renal tissue, visceral pain often accompanied by autonomic nerve reaction and low back pain caused by indwelling nephrostomy tube. ${ }^{4-6}$

Although the incidence rate of pain after percutaneous nephrolithotomy is not high, postoperative pain relief can not lead to discomfort in time, and cause additional factors such as delay of activity, pulmonary dysfunction and prolonged hospitalization. Although there are many analgesic factors, such as oral or intravenous sedatives, acupuncture, intercostal nerve block, tubeless PCNL, have been proved to reduce pain. However, there is not a perfect analgesic measure to implement. Tubeless PCNL may increase the risk of postoperative infection complications due to the lack of effective drainage. ${ }^{7}$ At the same time, different analgesic drugs have different adverse reactions in or out of the field. ${ }^{8}$ So it is very important to study the pain factors of PCNL. ${ }^{9}$ postoperative pain is a complex condition that needs a multimodal approach and good pain management can decrease hospital stay, rate of complications, and decrease overall health cost, but the causes and risk factors of pain after PCNL have rarely been reported. ${ }^{10}$ The purpose of this study was to screen the risk factors of moderate-to-severe pain after percutaneous nephrolithotomy, which was helpful to formulate effective prevention and treatment measures. 


\section{Methods}

\section{Clinical data}

According to the inclusion criteria, the clinical data of 331 patients who underwent PCNL in our hospital from September 2019 to February 2021 were retrospectively analyzed. In conformity with the occurrence of postoperative pain, patients were divided into mild or no pain group and moderate-to-severe pain group. The inclusion criteria were: 1) patients undergoing elective PCNL surgery under general anesthesia; 2) age 18-70 years; 3) ASA grade I or III; 4) Mallampati grade I-II; 5) body mass index (BMI) of $18-35 \mathrm{~kg} / \mathrm{m}^{2}$; and 6) signed the written informed consent.Exclusion criteria were:1)history of lipsilateral or bilateral kidney stones surgery;2)severe urinary tract infection;3)severe cardiac and pulmonary sufficiency and coagulation disorders;4) a spinal deformity, an underlying pathology, or prior spinal injury or surgery;5)neurological disorders, past or current substance abuse or other psychiatric diagnosis(fig. 1).

This study was approved by the medical ethics committee of our institutes and all patient subjects signed informed consent.According to the ordinary preoperative fasting guidelines, the patients fasted from both food and fluids for at least 8 hours before surgery.Intraoperative treatment A preventive antibiotic was given 30 minutes before surgery.Venous access was established in the upper extremities, and the blood pressure (BP), electrocardiogram (ECG), heart rate $(\mathrm{HR})$, and pulse oxygen saturation $\left(\mathrm{SpO}_{2}\right)$ were routinely monitored.

\section{Operation technique}


All the patients were under general anesthesia using the same type of medication. General anesthesia was induced with intravenous propofol ( $2 \mathrm{mg} / \mathrm{kg}$ ) plus sufentanil $(0.4 \mathrm{ug} / \mathrm{kg})$, and neuromuscular blockade was achieved with rocuronium $(0.6 \mathrm{mg} / \mathrm{kg})$ and maintained with intravenous and inhalational combined anesthesia.After endotracheal intubation, the mechanical ventilation was performed with tidal volume of $6 \sim 8 \mathrm{~mL} / \mathrm{kg}, 12$ breaths $/ \mathrm{min}$.Anesthesia was maintained with 1 minimum alveolar concentration (MAC) of sevoflurane (1.5\%-2.5\%) in a 60\% oxygen and air. Nitrous oxide was not used. Remifentanil infusions $(0.1-0.2 \mu \mathrm{g} / \mathrm{kg} / \mathrm{min})$ were used for analgesia. Expiratory $\mathrm{CO}_{2}, \mathrm{SpO}_{2}$, heart rate, and blood pressure were monitored continuously to ensure the maintenance of anesthesia.The MAP was maintained at 60-100 $\mathrm{mmHg}$, and the BIS at 40-60\%, these parameters being measured (and acted upon) every $5 \mathrm{~min}$ throughout the procedure.After proper padding of eyes, the patients were kept in prone position and all pressure points were secured.

Puncture: under the guidance of B-ultrasound, according to the distribution of stones, it is preferred to puncture the dorsal calyces with water or stones. The area between the posterior axillary line and the subscapular line was selected as the puncture point under 12 ribs or between 11 ribs. After the target calyces was punctured, the guide wire was placed to expand and the puncture time was recorded. Two step or balloon dilation can be used to establish the standard channel. Two steps: the first step is to use the F8-F16 fascial expander to expand gradually along the guide wire, and then put it into the sheath to establish the F16 
microchannel (fig.2). After ureteroscopic exploration, the position of the channel was determined and adjusted appropriately; the second step was to further expand to F24 with a telescopic metal dilator ( fig.3 ). Balloon dilation method: after the guide wire is successfully inserted, the catheter is pre expanded with the F8-F10 type fascial dilator, and then the F24 working sleeve is pre inserted into the back end of the balloon catheter, and the balloon is inserted into the target renal calyces under ultrasonic monitoring. After entering, the balloon is pressurized and filled. Ultrasound can clearly observe the double track image of balloon catheter dilatation. Then push the F24 jacket onto the target calyx. The balloon was removed and placed by indwelling guide wire, and the establishment of balloon channel was observed by nephroscope(fig. 4).

Lithotripsy: in this study, pneumatic ballistic lithotripsy was selected. Different stones can be operated with lithotripsy forceps, hanging basket and other instruments. Stones can be broken into small pieces and sucked out or rinsed with washing liquid. Slightly larger stones can be taken out with stone taking forceps. In the process of lithotripsy, the neck of calyces should be protected to avoid serious bleeding. The main measure is to determine the tilt angle of nephroscope according to the compliance of the kidney itself. In the non target calyceal surgery, the pressure of puncture should be paid attention to, and the relative fixation of nephroscope should be paid attention to in the process of lithotripsy. If no stone is detected, the channel can be re established according to the above steps to ensure a higher stone removal rate. B ultrasound was used to observe the residual stones after operation. 
Indwelling nephrostomy tube for 5-7 days after operation, according to the intraoperative and postoperative recovery, the extubation time should be appropriately advanced or extended. Ureteral stent should be retained for 2-4 weeks after operation. If ureteral stricture occurs, the duration of ureteral stent should be prolonged. If there is no fever or lumbar discomfort, the catheter can be removed after the nephrostomy tube is pulled out. ${ }^{11}$

Ondansetron $8 \mathrm{mg}$ was administered to all patients 20 minutes before the end of the surgery. After the procedure, the patients were sent to the recovery room and monitored continuously until full recovery.Residual neuromuscular blockade was antagonised with neostigmine and tropine when necessary.When patients achieved extubation criteria (awake and cooperative, fully rewarmed, haemodynamically stable, with blood gases within the normal ranges), extubation was accomplished.Patients were evaluated using the modified Aldrete scoring system(scoring $\geq 13$ ) until ready for discharge from the PACU .

\section{Pain assesment}

Postoperative pain was assessed using visual analogue scale (VAS), which was graded from 0 to 10 where 0 signified no pain whereas 10 signified most severe pain. In the preoperative period, patients were instructed on how to use VAS for pain. It was used to assess the degree of pain at $6,12,24$, and $48 \mathrm{~h}$ after surgery.If VAS scores exceeded 4/10 scores, rescue analgesia was intravenously administered according to a standardized 
institutional protocol for pain treatment until the pain was relieved to a level falling below a VAS score $<4$.The patients were divided into 2 groups based on this score: mild or no or no pain (VAS score 0-3), moderate-to-severe pain (VAS score 4-10).

Data collection is performed by research assistants for age,gender, body mass index (BMI),ASA grading,co-morbidities,number of renal calculus,renal calculus size,degree of hydronephrosis, preoperative urine culture,preoperative creatinine,stone burdens, expansion method,tract diameter, nephrostomy tube,calculi residual,operative position , puncture time ,number of expanded channels, expanded channel time, duration of surgery,vital signs and VAS pain score.

\section{Statistical analysis}

SPSS 23.0 software was used for data analysis. Measurement data are expressed as mean \pm standard deviation and were assessed by $t$ test. The Kolmogorov-Smirnoff single-sample test was used to calculate the normal distribution of continuous variables before doing further comparisons. The chisquare test was used to compare the 2 groups. When $\mathrm{P}$ was $<0.05$, the difference was regarded as statistically significant. Multivariate logistic analysis was used to analyze the statistical significance of the indicators. When $P<0.05$, the difference was regarded as significant. 


\section{Results}

In this study,A total of 331 patients in our single center were included in this study.221 patients with accompanying moderate-to-severe pain,the incidence was $66.77 \%($ moderate/severe=5/1).The results (table 1 ) showed that there were no significant differences in age $(P=0.705)$, gender $(P=0.709), \mathrm{BMI}(P=0.414)$, preoperative urine culture $(P=0.707)$, preoperative creatinine (umol/L)(P=0.540), dilation method $(P=0.079)$, channel diameter $(P=0.535)$, nephrostomy tube $(P=0.185)$, operative position $(P=0.142)$ and puncture time $(P=0.235)$ between the two groups. However, there were significant differences in number of renal calculus $(P<0.01)$, renal calculus size $(P<0.01)$, degree of hydronephrosis $(P<0.01)$,ASA grading $(P=0.016)$, stone burdens $(P<0.01)$, calculi residual $(P<0.01)$,Number of expanded channels $(P=0.009)$, expanded channel time $(P=0.037)$, duration of surgery $(P=0.01)$. Multivariate logistic analysis (table 2$)$ showed that number of renal calculus $(\mathrm{OR}=5.303, P=0.001)$, stone burdens $(\mathrm{OR}=7.623, P=0.001)$, calculi residual $(\mathrm{OR}=0.2377, P=0.001)$ and duration of surgery $(\mathrm{OR}=1.024, P=0.001)$ were independent risk factors for moderate-to-severe postoperative pain after PCNL and are important indicators for clinical treatment.

\section{Discussion}

Renal calculi are mineral deposits in the renal collecting system that are free or attached to the renal papilla. In terms of composition, calcium oxalate and calcium phosphate account for about $8 / 10$. In recent 
years, the incidence rate of stones has increased, and the recurrence rate is up to half in the first 5 years of the first stone attack. Obesity, diabetes, hypertension, metabolic syndrome and stones form a vicious circle. These diseases increase the possibility of stones, and the occurrence of stones leads to the deterioration of hypertension, kidney disease and other diseases. At present, the treatment of renal calculi with clinical manifestations is mostly minimally invasive treatment, so as to reduce the surgical complications, reduce the surgical injury, improve the stone clearance rate, and achieve a higher quality of life. ${ }^{12-14} \mathrm{PCNL}$ is considered to be the first line of treatment for large renal calculi, and plays an important role in the treatment of particularly large $(>2 \mathrm{~cm})$ renal calculi and / or staghorn renal calculi. However, PCNL technology is very difficult, which is greatly affected by the composition, size and location of stones. At the same time, the number of stones, stone load, and the degree of hydronephrosis also affect the results of PCNL. Similarly, PCNL also has potential complications, such as bleeding, pain and so on. Septic shock and renal failure often occur in severe cases. ${ }^{15}$ Studies have shown that the causes of pain after PCNL include direct injury of renal cortex, high intrapelvic pressure (RPP), postoperative perirenal distension caused by extravasation of water, damage of collecting system caused by lithotripsy, and low back pain caused by indwelling nephrostomy tube.At present, there are few reports on the causes and risk factors of pain after PCNL. This study summarized and analyzed the clinical data of 331 patients to determine the risk factors and to provide a reference for clinical prevention.

In this study, we found that there were no significant differences in age,gender,BMI,preoperative 
urine culture, preoperative creatinine, dilation method, channel diamete, nephrostomy tube,operative position and puncture time between the mild or no or no pain group and the moderate-to-severe pain group $(P>0.05)$. However, there were significant differences in number of renal calculus,renal calculus size, degree of hydronephrosis,ASA grading,stone burdens,calculi residual,Number of expanded channels,expanded channel time,duration of surgery $(P<0.05)$.Multivariate logistic regression analysis showed that the independent risk factors for pain after PCNL included number of renal calculus,stone burdens,calculi residual and duration of surgery. Renal calculi originate from Randall's plaques of calyces. The more the number of stones, the more the number of involved calyces, or there are stones in both calyces and pelvis. For patients with multiple renal calyceal calculi, the scope of operation will increase. In the process of operation, it is very easy for the surgeons to touch the collecting system when they put the gravel probe rod from one renal calyceal to another adjacent renal calyceal. If they are careless, the collecting system will be damaged, resulting in bleeding and postoperative pain. Due to bleeding, blurred vision will aggravate this process. Renal calculi originate from Randall's plaques of renal calyces and crystallize with minerals in renal pelvis or renal calyces. As time goes on, the volume of renal calculi increases gradually and eventually forms partial or complete staghorn calculi. For patients with large burden of such calculi, the operation time is relatively long, the intraoperative bleeding is more, and the postoperative pain is heavier. For patients with large preoperative stone load, large number of stones, and partial or complete staghorn stones, the larger the complex stones, the lower the corresponding 
stone clearance rate, the greater the difficulty coefficient of operation, the longer the operation time, the more blood loss during operation, and the more severe the postoperative pain. At the same time, residual stones can be discharged from the ureter. In the process of stone removal, irregular stones may scratch the ureteral mucosa, causing pain or obstruction, leading to colic. Meanwhile, studies have shown that the incidence rate and duration of postoperative pain will increase significantly when the intraoperative pressure is greater than $30 \mathrm{mmhg}$. In all PCNL operations, the flow velocity is $200-350 \mathrm{ml} / \mathrm{min}$, and the pressure of renal pelvis is less than or equal to $2.94 \mathrm{kPa}(30 \mathrm{cmH} 2 \mathrm{O})$, which reduces the research bias caused by intrarenal pressure. ${ }^{16}$

Anatomical studies have shown that the main sources of acute pain after PCNL are visceral pain from kidney and ureter and body surface pain from incision. Renal pain comes from T10-L1 spinal nerve, and ureteral pain comes from T10-L2 spinal nerve. However, the incision and path usually formed under the 12th rib or between the 10th and 11th rib, and the cutaneous innervation in this area was mainly undertaken by T10-11. The indwelling of nephrostomy tube, peritubular compression of renal cortex and dilatation of renal capsule can also aggravate postoperative pain. However, the discomfort, pressure and pain caused by nephrostomy increase the importance of postoperative analgesia.

Inadequate analgesia can result in increased morbidity with delayed, mobilization, impaired ventilation, and prolonged hospitalization, which may increase the overall cost of the procedure. To lower the morbidity of 
PCNL, proper and adequate management of postoperative pain remain an integral component of PCNL.There are many ways of reducing postoperative pain.These include local anesthetic pressure during surgery, paravertebral block (T11-L1), erector spinae plane block,low thoracic paravertebral block, paravertebral block,quadratus lumborum block III,intercostal nerve block, using postoperative opioid analgesia, reducing the size of nephrostomy tube or not placing a nephrostomy tube at all. ${ }^{17-23}$

There are some limitations of our study. Firstly, it was not a prospective randomized controlled trial. Further larger-sample long-term follow-up is needed to obtain more accurate clinical data for in-depth analysis. Secondly, the pathophysiology of pain after PCNL has not been elaborated and explored in detail. In addition, this was a single center regression study, and multi-center studies can obtain more accurate clinical reference data.

\section{Conclusions}

Based on our findings, independent risk factors for pain after PCNL include number of renal calculus, stone burdens,calculi residual and duration of surgery. Early identification of the risk factors for renal atrophy moderate-to-severe postoperative pain after PCNL and formulation of reasonable preventive measures are needed to control the occurrence of the pain.

\section{Abbreviations}


ASA: American Society of Anesthesiologists ;BMI:body mass index;BP:blood

pressure;ECG:electrocardiogram;HR:heart rate ;MAC:minimum alveolar concentration ;PCNL: percutaneous

nephrolithotomy ;RPP:high intrapelvic pressure ;SpO 2 pulse oxygen saturation

\section{Acknowledgements}

This research was supported by the department of Anesthesiology of Beijing Tsinghua Changgung Hospital and

school of clinical medicine of Tsinghua University

\section{Authors' contributions}

Study design: HTW, TFD, HZ. Study conduct: HTW, TFD, HZ. Data analysis: HTW, TFD. Writing paper: HTW, TFD,

HZ. All authors read and approved the final manuscript version.

Funding

Financial support for this study was provided, in part, by departmental funding.

\section{Availability of data and materials}

The raw data of this study are available from the corresponding author on reasonable request.

\section{Declarations}

\section{Ethics approval and consent to participate}

This study was approved by ethics committee of Beijing Tsinghua Changgung Hospital.All patients 
signed the informed consent.The procedures did not require any additional intervention, and all data were analyzed anonymously. All methods were carried out in accordance with relevant guidelines and regulations.

\section{Consent for publication}

Not applicable.

\section{Competing interests}

The authors declare that they have no competing interests.

\section{References}

1. Sio M D, Manfredi C, Fusco F, et al. Recent advances in percutaneous lithotripsy techniques[J]. Current Opinion in Urology, 2020, Publish Ahead of Print(1). doi:10.1097/MOU.0000000000000829.PMID:33196538

2. Kyriazis I, Panagopoulos V, Kallidonis P, Özsoy M, Vasilas M, Liatsikos E. Complications in percutaneous nephrolithotomy. World J Urol. 2015 Aug;33(8):1069-77. doi: 10.1007/s00345-014-1400-8. Epub 2014 Sep 14. PMID: 25218854.

3. Ugras MY, Toprak HI, Gunen H, Yucel A, Gunes A. Instillation of skin, nephrostomy tract, and renal puncture site with ropivacaine decreases pain and improves ventilatory function after percutaneous 
nephrolithotomy. J Endourol. 2007 May;21(5):499-503. doi: 10.1089/end.2006.0335. PMID: 17523902.

4. Dam M, Hansen CK, Poulsen TD, Azawi NH, Wolmarans M, Chan V, Laier GH, Bendtsen TF, Børglum J. Transmuscular quadratus lumborum block for percutaneous nephrolithotomy reduces opioid consumption and speeds ambulation and discharge from hospital: a single centre randomised controlled trial. Br J Anaesth. 2019 Aug;123(2):e350-e358. doi: 10.1016/j.bja.2019.04.054. Epub 2019 May 30. PMID: 31153628; PMCID: PMC6676058.

5. Alsyouf M, Abourbih S, West B, Hodgson H, Baldwin DD. Elevated Renal Pelvic Pressures during Percutaneous Nephrolithotomy Risk Higher Postoperative Pain and Longer Hospital Stay. J Urol. 2018 Jan;199(1):193-199. doi: 10.1016/j.juro.2017.08.039. Epub 2017 Aug 12. PMID: 28807646.

6. Capodice JL, Parkhomenko E, Tran TY, Thai J, Blum KA, Chandhoke RA, Gupta M. A Randomized, Double-Blind, Sham-Controlled Study Assessing Electroacupuncture for the Management of Postoperative Pain after Percutaneous Nephrolithotomy. J Endourol. 2019 Mar;33(3):194-200. doi: 10.1089/end.2018.0665. PMID: 30693806.

7. Chang $\mathrm{CH}$, Wang $\mathrm{CJ}$, Huang SW. Totally tubeless percutaneous nephrolithotomy: a prospective randomized controlled study. Urol Res. 2011 Dec;39(6):459-65. doi: 10.1007/s00240-011-0363-0. Epub 2011 Feb 18. PMID: 21331773.

8. Singh P, Yadav S, Singh A, Saini AK, Kumar R, Seth A, Dogra PN. Systemic Inflammatory Response 
Syndrome Following Percutaneous Nephrolithotomy: Assessment of Risk Factors and Their Impact on Patient Outcomes. Urol Int. 2016;96(2):207-11. doi: 10.1159/000441954. Epub 2016 Jan 9. PMID: 26745881.

9. Feng D, Tang Y, Bai Y, Wei W, Han P. The efficacy of local anesthetic infiltration around nephrostomy tract in alleviating postoperative pain after percutaneous nephrolithotomy: A network meta-analysis. Asian J Surg. 2020 Jun;43(6):702-704. doi: 10.1016/j.asjsur.2020.01.007. Epub 2020 Jan 29. PMID: 32007369.

10. Gadzhiev N K G , Obidnyak V M O, Gorelov D S G , et al. Complications after PCNL: diagnosis and management[J]. Urologiia (Moscow, Russia: 1999), 2020, 5_2020:139-148.PMID: 33185362.

11. 李建兴,刘宇保,肖博.经皮肾镜碎石术安全共识[J].现代泌尿外科杂志,2020,25(04):296-301.

12. Khan SR, Pearle MS, Robertson WG, Gambaro G, Canales BK, Doizi S, Traxer O, Tiselius HG. Kidney stones. Nat Rev Dis Primers. 2016 Feb 25;2:16008. doi: 10.1038/nrdp.2016.8. PMID: 27188687; PMCID: PMC5685519.

13. Romero V, Akpinar H, Assimos DG, authors. Kidney stones: A global picture of prevalence, incidence and associated risk factors. Rev Urol. 2010;12:e86-96.PMID: 20811557

14. Ghani KR, Andonian S, Bultitude M et al: Percutaneous nephrolithotomy: Update, trends, and future directions. Eur Urol, 2016; 70(2): 382-96 dol:10.1016/j.eururo.2016.01.047. PMID: 26876328.

15. Karalar $\mathrm{M}$, Tuzel $\mathrm{E}$, Keles I et al: Effects of parenchymal thickness and stone density values on 
percutaneous nephrolithotomy outcomes. Med Sci Monit, 2016; 22: 4363-68.

dol10.12659/msm.898212.PMID: 27842051.

16. Alsyouf $M$, Abourbih S, West B, Hodgson H, Baldwin DD. Elevated Renal Pelvic Pressures during Percutaneous Nephrolithotomy Risk Higher Postoperative Pain and Longer Hospital Stay. J Urol. 2018 Jan;199(1):193-199. doi: 10.1016/j.juro.2017.08.039. Epub 2017 Aug 12. PMID: 28807646.

17. Chin KJ, Adhikary S, Sarwani N, Forero M, authors. The analgesic efficacy of pre-operative bilateral erector spinae plane (ESP) blocks in patients having ventral hernia repair. Anaesthesia. 2017;72:452-60. dol:10.1111/anae.13814.PMID: 28188621

18. Prasad M, Varshney R, Jain P, et al. Postoperative analgesic efficacy of fluoroscopy-guided erector spinae plane block after percutaneous nephrolithotomy (PCNL): A randomized controlled study[J]. Saudi Journal of Anaesthesia, 2020,14(4):480. dol: 10.4103/sja.SJA_26_20.PMID: 33447190.

19. Yayik Ahmet Murat,Ahiskalioglu Ali,Demirdogen Saban Oguz et al. Ultrasound-guided low thoracic paravertebral block versus peritubal infiltration for percutaneous nephrolithotomy: a prospective randomized study.[J] .Urolithiasis, 2020, 48: 235-244. dol:10.1007/s00240-018-01106-w.PMID: 30564847.

20. Baldea KG,Patel PM,Santos GD,et al.Paravertebral block for percutaneous nephrolithotomy: a prospective, randomized, double-blind placebo-controlled study[J]. World Journal of Urology, 2020(5). 
dol:10.1007/s00345-020-03093-3.

21. Korgün kmen, Kmen BM.Ultrasound-guided anterior quadratus lumborum block for postoperative pain after percutaneous nephrolithotomy: a randomized controlled trial[J]. Korean Journal of Anesthesiology, 2020, 73(1). dol:10.4103/aer.AER_45_20.

22. Chen T,Zhu ZQ,Du J.Efficacy of Intercostal Nerve Block for Pain Control After Percutaneous Nephrolithotomy: A Systematic Review and Meta-Analysis[J]. Frontiers in Surgery, 2021, 8. dol:10.3389/fsurg.2021.623605.PMID: 33585552.

23. Shariat Moharari Reza,Valizade Ali,Najafi Atabak et al. Analgesic Efficacy of Nephrostomy Tract inFiltration of Bupivacaine and Ketamine after Tubeless Percutaneous Nephrolithotomy:A Prospective Randomized Trial.[J] .Iran J Pharm Res, 2016, 15: 619-26.PMID: 27642334. 
Fig.1. Study identification and selection procedure 
Fig.2. Under the guidance of ultrasound, the puncture needle selected the puncture point between the 11th intercostal or 12th intercostal posterior axillary line and the subscapular angle to puncture into the target calyces and establish the $16 \mathrm{~F}$ microchannel. 
Fig.3. The "two-step" method was used to establish the standard channel. First, a small ureteroscope was used to enter the microchannel to observe the position of the channel, and the preliminary adjustment was made. After the adjustment, the sheath was gradually expanded to establish the $24 \mathrm{~F}$ standard channel 
Fig.4. The puncture needle was punctured to the target calyces, and then the guide wire was indwelling and expanded along the guide wire to establish the skin kidney channel. The ultrasound pneumatic lithotripsy was used. One side of the lithotripsy was performed, and the negative pressure suction was performed at the same time. Small stones could be sucked out. 


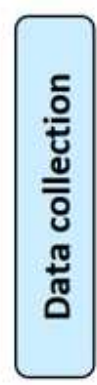

Recordings identified through database searching from September 2019 to February 2021

$(n=1081)$

The inclusion criteria were: 1) patients undergoing elective PCNL surgery under general anesthesia; 2) age 18-70 years; 3) ASA grade I or III; 4) Mallampati grade $1-I I$; 5 ) body mass index (BMI) of $18-35 \mathrm{~kg} / \mathrm{m}^{2}$; and 6 ) signed the written informed consent

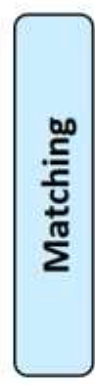
(VAS score 4-10)group $(n=220)$

Patients excluded $(n=705)$

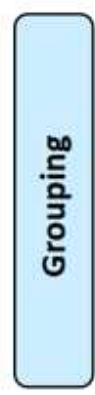

Patients enrolled

$(n=331)$

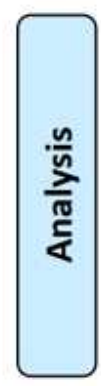

mild or no pain (VAS score

0 -3) group

$(n=110)$

Statistical analysis, obtain the data

\section{Figure 1}

Study identification and selection procedure 


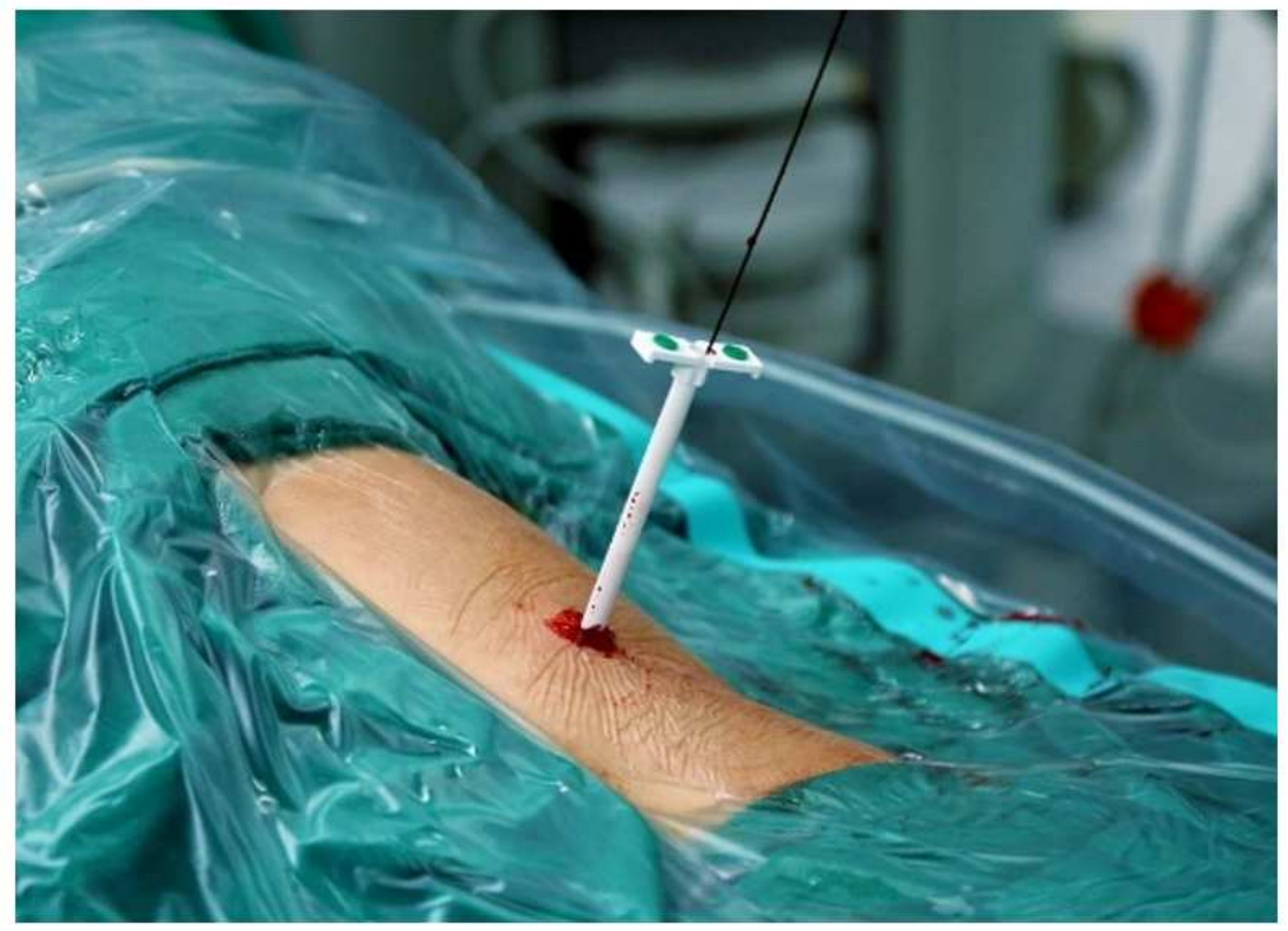

\section{Figure 2}

Under the guidance of ultrasound, the puncture needle selected the puncture point between the 11th intercostal or 12th intercostal posterior axillary line and the subscapular angle to puncture into the target calyces and establish the $16 \mathrm{~F}$ microchann 


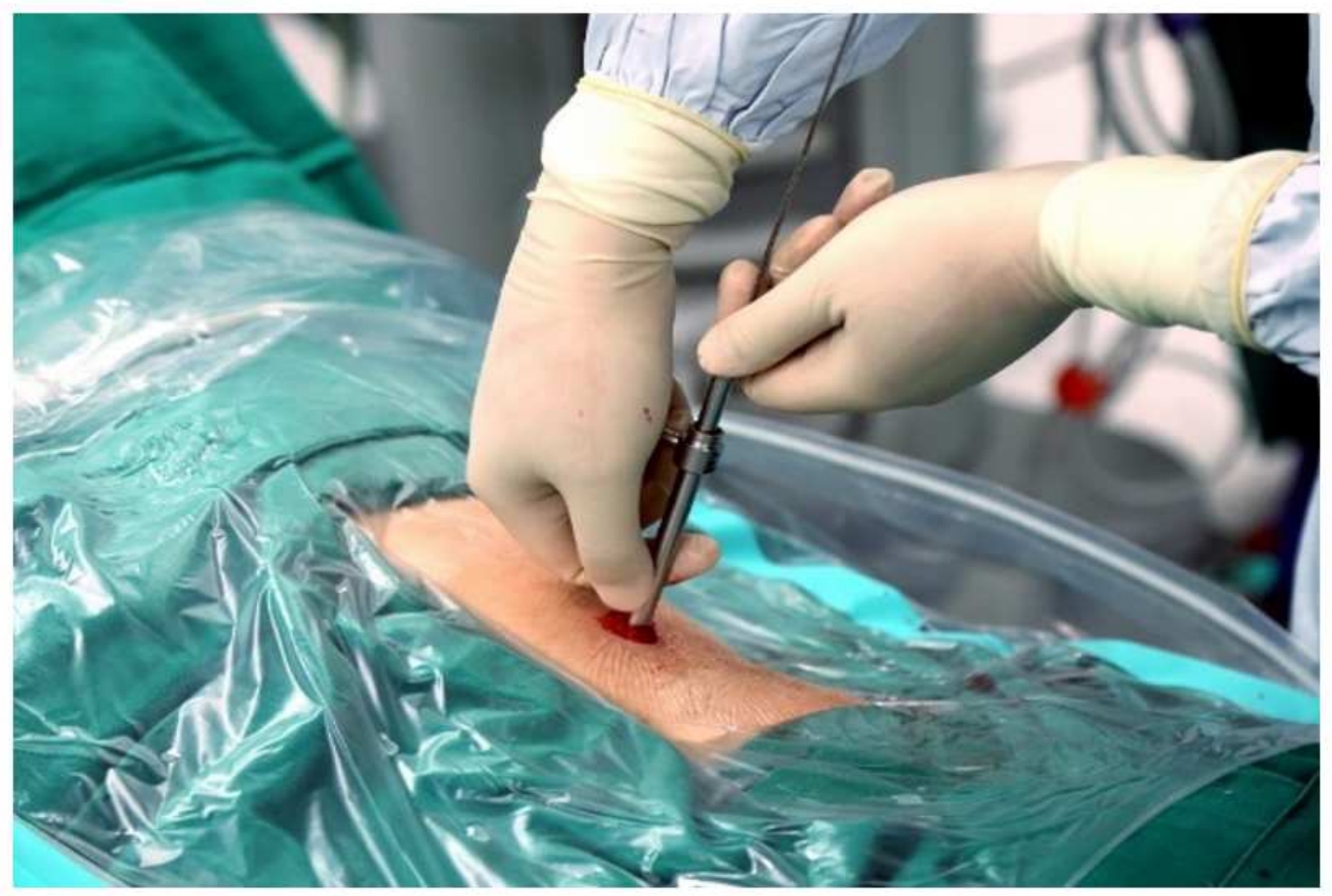

\section{Figure 3}

The "two-step" method was used to establish the standard channel. First, a small ureteroscope was used to enter the microchannel to observe the position of the channel, and the preliminary adjustment was made. After the adjustment, the sheath was gradually expanded to establish the $24 \mathrm{~F}$ standard channel 

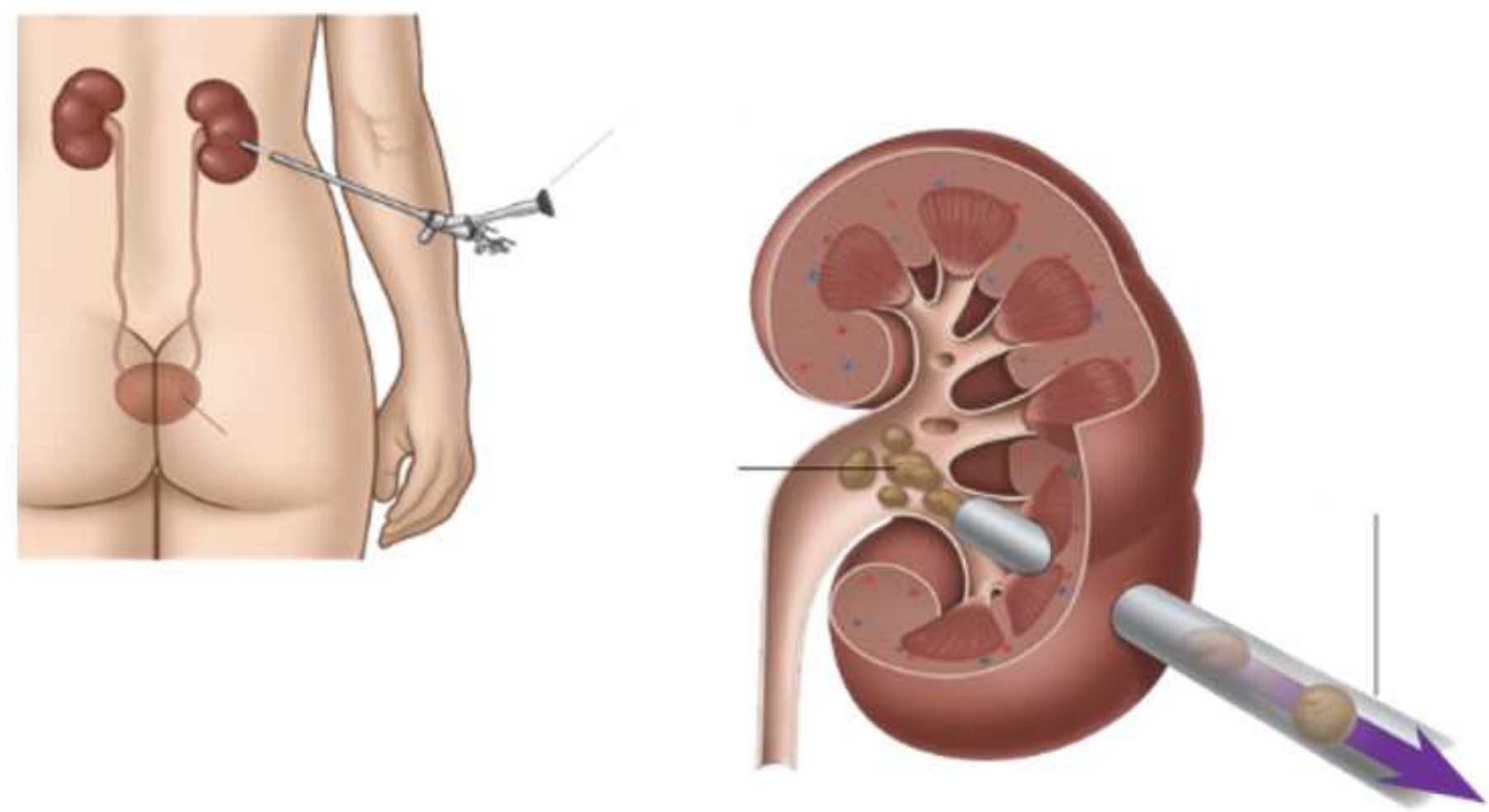

Figure 4

The puncture needle was punctured to the target calyces, and then the guide wire was indwelling and expanded along the guide wire to establish the skin kidney channel. The ultrasound pneumatic lithotripsy was used. One side of the lithotripsy was performed, and the negative pressure suction was performed at the same time. Small stones could be sucked out 